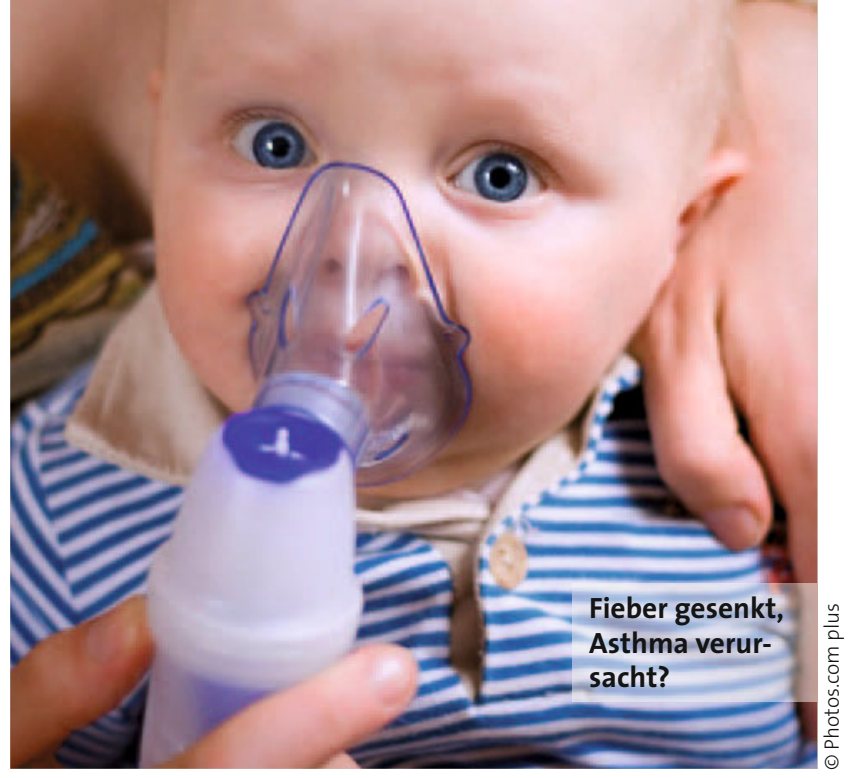

\title{
Paracetamol erhöht das Asthmarisiko
}

\section{Als Fiebersenker und als Schmerzmittel wird bei Kindern bevorzugt Paracetamol eingesetzt. Möglicherweise muss man mit der Gabe dieses Medikaments etwas vorsichtiger sein.}

— In diese weltweite Untersuchung wurden 322959 Kinder im Alter von 13-14 Jahren eingeschlossen. Das Ziel war es, zu eruieren, ob die Einnahme von Paracetamol das Risiko für Asthma erhöht. Zu diesem Zweck wurde die Einnahme dieses Medikaments während des letzten Jahres korreliert mit derzeitig bestehenden Asthmasymptomen.

Die Auswertungen zeigten eine signifikante, dosisabhängige Risikoerhöhung. Bei höheren Paracetamoldosierungen war es mehr als verdoppelt. Ebenso fand sich nach Paracetamoleinnahme eine Erhöhung des Risikos für Ekzem und Rhinokonjunktivitis. Die Autoren meinen daher, dass die Paracetamoleinnahme einen bedeutsamen Risikofaktor für Asthma, Rhinokonjunktivitis und Ekzem sein könnte.

\section{Kommentar}

Paracetamol hat den Ruf, weitestgehend risikofrei zu sein. Deswegen wird es auch gerne Kindern verabreicht. Die vorliegenden Daten, die vor allem durch die enorme Fallzahl bestechen, implizieren, dass dieser Ruf vielleicht nicht ganz berechtigt ist. Der Verdacht, dass ein Zusammenhang zwischen der Paracetamoleinnahme und Asthma bestehen könnte, war zunächst in epidemiologischen Untersuchungen aufgetaucht. Inzwischen hat er sich wiederholt auch in klinischen Studien bestätigt. Falls der Zusammenhang tatsächlich kausaler Natur sein sollte, hätte er enorme Bedeutung. Bis zur letztlichen Klärung aller Details, sollten wir vielleicht beim Verabreichen von Paracetamol speziell bei Kindern größere Zurückhaltung walten lassen.

E. ERNST =

- R. W. Beasley et al.

Acetaminophen use and risk of asthma, rhinoconjunctivitis and eczema in adolescents: ISAAC phase three. Am. J. Respir. Crit. Care Med. 2010; doi: 10.1164/rccm.201005-0757OC(accepted for publication). 